\title{
Notwehrbefugnis und Grundrechte
}

\author{
Jürgen Schwabe*
}

A. Rätselhafte deutsche Strafrechtswissenschaft ........................ 218

B. Wissenschafts-Echo: Fehlanzeige..... 219

C. Die Auffassung Engländers zur Grund-

rechtskontrolle der Notwehr......... 220

I. Zur Eingriffsermächtigung .......... 221
II. Zur Duldungspflicht ....

D. Fazit............................ 226

E. Von der Zoomaufnahme zum Weitwinkel

\section{A. Rätselhafte deutsche Strafrechtswissenschaft}

Angenommen, ein Jurist aus einem anderen Erdteil käme zur Fortbildung nach Europa, studierte ein Jahr in Österreich und reiste dann nach Deutschland weiter.

Hier interessiert ihn das Strafrecht und insbesondere die Rechtfertigung durch Notwehr. Nach den ersten Studien erbittet er von einem deutschen Professor einen Rat: „Ich möchte etwas über die Grenzen des Notwehrrechts erfahren. Dazu habe ich alle Lehrbücher und natürlich auch Ihres, Herr Professor, durchgelesen, außerdem neun Kommentare, eine Monographie von Wittemann über „Grundlinien und Grenzen der Notwehr in Europa" ${ }^{1}$ sowie eine größere Anzahl von Aufsätzen. Überall wird die Vereinbarkeit des Notwehrrechts mit der EMRK diskutiert. Das leuchtet mir von meinen Studien in Österreich ein, wo die Konvention Verfassungsrang hat. Aber bei meiner Lektüre bin ich nirgendwo auf eine Beurteilung des Notwehrrechts nach der deutschen Verfassung gestoßen. Offenbar gibt es eine Norm, wonach die Grundrechte nicht für das Strafrecht gelten. Aber welche Norm ist das, bitte?“ „Es gibt keine solche Norm!“ „Und weshalb wird dann die Vereinbarkeit des Notwehrrechts nur mit der EMRK und nicht mit den Grundrechten der Verfassung geprüft?“

Weiterhin angenommen, der Befragte habe ein paar Gläschen Wein genossen und gerade eine launige Phase, könnte er mit dem Fürsten Orlofsky aus der „Fledermaus“ antworten:

„Ach, fragen Sie nicht, bitte, warum ich das denn tu, 's ist mal bei uns so Sitte, chacun à son goût!"

Ein paar wenige halten sich nicht an die Sitte, wie der Ausländer bei weiteren Recherchen ermittelt. In einem Kommentar ${ }^{2}$ findet er ein paar dürftige Hinweise auf die Grundrechte, eine ausführlichere Diskussion zu grundrechtlichen Schutzpflichten in einem Aufsatz von Bernsmann ${ }^{3}$ und in einer Dissertation von Lührmann, ${ }^{4}$ schließ-

* Prof. Dr. Jürgen Schwabe lehrte öffentliches Recht an der Universität Hamburg.

1 F. Wittemann, Grundlinien und Grenzen der Notwehr in Europa, Frankfurt/M 1997.

2 H.L. Günther, in: Rudolphi/Horn (Hrsg.), Systematischer Kommentar zum StGB, $\mathbb{3} 32$, Rn.11.

3 W. Bernsmann, Tödliche Notwehr, ZStW 104(1992), S. 290 (307).

4 O. Lührmann, Tötungsrecht zur Eigentumsverteidigung, Frankfurt/M 1999. 
lich eine umfassende Darstellung des Problems in der 2008 erschienenen Habilitationsschrift von Engländer. ${ }^{5}$ Aus der zweiten und der vierten dieser Quellen ergebe sich, so berichtet er später seinem Professor, dass bereits 1974 die Maßstäblichkeit der Grundrechte als Abwehrrechte für die Notwehrbefugnis eingefordert wurde. ${ }^{6} \mathrm{Ob}$ es denn für den wissenschaftlichen Diskurs in Deutschland normal sei, dass sich erst nach 34 Jahren jemand mit einer These auseinander setze? Oder ob sich, deren Absurdität wegen, eine Auseinandersetzung gar nicht lohne, was nun von Engländer verkannt worden sei?

In diesem Augenblick wird der befragte Strafrechtsprofessor zu einem wichtigen und länger andauernden Telefongespräch gerufen. Auf die gestellte Frage ist er später nie mehr zurückgekommen, und dem ausländischen Juristen schien es geboten, sich nicht durch eine Erinnerung unbeliebt zu machen.

\section{B. Wissenschafts-Echo: Fehlanzeige}

Die Abhandlung von 1974 ist - nach einer wenig förderlichen Replik von Kratzsch ${ }^{7}$ im selben Jahr - bis zu der Schrift von Engländer in der Sache völlig unbeachtet geblieben. ${ }^{8}$ Ganz selten verirrte sie sich in ein Literaturverzeichnis, ohne aber im Text erwähnt zu werden. ${ }^{9}$ E. Dreher vermerkte 1975 in der 35.Auflage des Beck'schen Handkommentars in Rdnr. 21: „Zu den Schranken, die Art. 2 II GG dem $\$ 32$ ziehen soll, Schwabe..."; diese Notiz hielt sich immerhin 31 Jahre. ${ }^{10}$

18 Jahre nach der Veröffentlichung schrieb Bernsmann: ${ }^{11}$ „Das Notwehrrecht zu töten ist bislang noch kaum einer ausführlicheren verfassungsrechtlichen Kontrolle unterzogen worden. Eine Ausnahme macht Schwabe, NJW 1974, 670.“ Jedoch wird in seiner Befassung mit Art. 2 II GG, nur eine Seite später, der (Ausnahme-)Aufsatz nicht mehr zitiert und auch auf den restlichen 20 Seiten in der Sache mit keiner Silbe erwähnt. Die dort allein diskutierte Abwehrfunktion der Grundrechte kommt bei Bernsmann überhaupt nicht vor, vielmehr befasst er sich ausschließlich mit der grundrechtlichen Schutzpflicht.

5 A. Engländer, Grund und Grenzen der Nothilfe, Tübingen 2008.

6 J. Schwabe, Grenzen des Notwehrrechts, NJW 1974, S. 670.

7 D. Kratzsch, Verfassungsbeschwerde gegen fehlerhafte Anwendung des $\$ 53$ StGB?, NJW 1974, S. 1546.

8 Nicht nur in strafrechtlichen Abhandlungen, über die nachfolgend berichtet wird. Die Vereinbarkeit eines exzessiven Notwehrrechts mit Grundrechten wird in keinem Staatsrechtslehrbuch (einschließlich des Monumentalwerks von K. Stern und dem Handbuch des Deutschen Staatsrechts) thematisiert und in keinem der zehn wichtigsten GG-Kommentare mit Ausnahme relativ vager Andeutungen von $U$. di Fabio, in Maunz/Dürig, Grundgesetz, München Loseblatt (Stand 4/2004), Art. 2 II 1 Rn. 37.

9 In den Lehrbüchern von R. Maurach/H.Zipf, Strafrecht AT, 5.Aufl. Heidelberg 1977,S. 372, und C.Roxin, Strafrecht AT I, 4.Aufl. München 2006, in $\$ 15$, sowie im MK-StGB (Erb) und im Schönke/ Schröder (Lenckner/Perron).

10 H.Tröndle hat sie stetig beibehalten, T. Fischer aber in der 53. Auflage 2006 gelöscht.

11 Notwehr (Fn. 3). 
Exakt ebenso verfährt Olivia Lührmann. ${ }^{12}$ Grundrechte bestehen offenbar ausschließlich aus Schutzpflichten: So beginnt auf S. 69 innerhalb des Abschnitts „D. Verfassungsrechtliche Probleme des Notwehrrechts“ der Unterabschnitt „II. Tötungsrecht zur Sachwertverteidigung und Art. 2 Abs. 2 i.V. mit Art. 1 GG“ wie folgt:

„In der Gestattung eines Notwehrrechts, das die Tötung des Angreifers zur Verteidigung von... Sachwerten zulässt, könnte ein Verstoß gegen Art.1 I i.V.m. Art. 2 II 1 GG zu sehen sein.

\section{Schutzpflichtenkollision. ${ }^{13}$}

Das Entstehen einer Notwehrlage führt zu einer Berührung staatlicher Schutzpflichten."

... was dann über viele Seiten diskutiert wird. Grundrechte des status negativus kommen nicht vor. Sie wären offenbar auch entbehrlich, wenn man den tödlichen Schusswaffeneinsatz der Polizei zu beurteilen hätte. Wie die sachlich ignorierte Veröffentlichung von 1974 ins Literaturverzeichnis der Dissertation geriet, bleibt ein ebensolches Rätsel ${ }^{14}$ wie die Verfahrensweise bei Bernsmann.

1996 habilitierte Otto Lagodny über „Strafrecht vor den Schranken der Grundrechte“, doch war ihm trotz Bernsmanns Erinnerung der Aufsatz von 1974 entgangen. Engländer hingegen hat ihn entdeckt und sich mit ihm auseinander gesetzt, allerdings ohne jede Verwunderung, dass er der erste ist. Wie es um die Güte seiner Argumente steht, sei im Folgenden dargelegt.

\section{Die Auffassung Engländers zur Grundrechtskontrolle der Notwehr}

Ich messe das Notwehrrecht an den Grundrechten als Abwehrrechten, weil staatliches Recht den Angegriffenen zu einem Eingriff ermächtigt und dem Angreifer eine Duldungspflicht auferlegt. Gleiches gilt beispielsweise für die Festnahmebefugnis Privater nach $\$ 127$ I 1 StPO. Dass diese grundrechtskonform ist, spielt keine Rolle, es geht um die Maßstäblichkeit der negatorischen Grundrechte. Praktische Bedeutung würden sie erlangen, wenn man die Befugnis etwas variiert, sie beispielsweise auch für Ordnungswidrigkeiten gelten ließe oder -für den Fall einer längeren Säum-

12 Tötungsrecht (Fn. 4).

13 Von einer solchen Kollision, bei der auch Bernsmann endet, kann man bestenfalls vor irgend einer staatlichen Regelung sprechen, nach einer Setzung von Recht unterliegt dieses mindestens auf der Opferseite, bei einem (Teil-) Verbot der Gegenwehr, dem grundrechtlichen status negativus. Zutreffend Engländer (Fn. 5), S. 351, indes mit dem falschen Schluss, es würden „nicht lediglich zwei Schutzrechte miteinander in Konflikt stehen, sondern zudem ein Schutzrecht mit einem Abwehrrecht", also offenbar insgesamt 3 Grundrechtspositionen bei 2 Beteiligten!

14 Mit vergleichbaren Rätseln habe ich mich in JZ 2004, 393 („, Ins Horn gezwickt“) befasst. Zu dem dort diskutierten Faktor der Informationsüberlastung sei noch auf H. Beste/W. Kroeber-Riel/H. Jung, Auswirkungen der zunehmenden Informationsüberlastung auf das Verhalten von Juristen, in: Fiedler, (Hrsg). Verfassungsrecht und Völkerrecht, Gedächtnisschrift für W.K.Geck, Köln 1989, S. 377 ff., hingewiesen. 
nis der Polizei - mit einer Ermächtigung zur Haft verknüpfen würde. ${ }^{15}$ Weshalb gelten unbestrittener Maßen die negatorischen Grundrechte, sobald man den Ermächtigten (eventuell: gerade noch und umstritten) als Beliehenen bezeichnen kann, aber nicht mehr ohne diese Qualifizierung?

Engländer hält die Eingriffsermächtigung ebenso wie die Duldungsverpflichtung für irrelevant.

\section{Zur Eingriffsermächtigung}

„Nur weil der Staat einem Privaten einer Handlung erlaubt, d.h. nicht verbietet, ist er noch nicht an der Vornahme dieser Handlung beteiligt. Ansonsten müsste jedes nicht verbotene Verhalten einer Privatperson ... als grundrechtsrelevanter Eingriff aufgefasst werden. Alles zulässige private Handeln wäre der staatlichen Sphäre zuzurechnen und damit den für diese geltenden Begrenzungen unterworfen." 16

1. Diese Argumentation krankt am Mangel begrifflicher Präzision. Engländer spricht hier von „Handlungen“ und „Verhalten“ und verdeckt damit in unangemessener Weise, was er nur fünf Zeilen zuvor zutreffend als maßgeblich bezeichnet hat: „die Beeinträchtigung oder Beseitigung grundrechtlich geschützter Güter“. Wenn man das weglässt, gelangt man schnell zu der Unsinnsthese, alles erlaubte/nicht verbotene Privathandeln, also auch das Pfeifen auf einem Spaziergang oder die Einladung zu einem Essen, sei dem Staat zuzurechnen. ${ }^{17}$

15 Weil auch Fälle, die es aktuell nicht (mehr) gibt, ein dogmatisches Problem erhellen können, darf man sich auch eine Ermächtigung vorstellen, einen unwilligen Schuldner in Schuldhaft zu nehmen.

16 Engländer, Nothilfe (Fn. 5), S. 181.

17 Derlei Einfalt ist mir gelegentlich unterstellt worden. R. Alexy, Theorie der Grundrechte, Frankfurt 1986, S. 417, den Engländer zitiert, hat mit Recht darauf hingewiesen, dass dergleichen nicht gemeint sein könne. Aber man wird (beispielsweise bei G. Robbers, Sicherheit als Menschenrecht, Baden-Baden 1987, S. 128) dem umwerfenden Einwand konfrontiert „Wenn der Staat den Ehebruch nicht verbietet, so ist er doch nicht erlaubt und er kann dem Staat nicht zugerechnet werden. " Trefflich! Und noch weniger ist es ein Staatsakt, wenn der Schüler Fritz dem Franz die Freundin ausspannt. Da es aber selbstverständlich nur um die Erlaubnis für „Eingriffe in Grundrechtsgüter“ (so Robbers in der zweiten Zeile derselben Seite) geht und Art. 6 GG (entgegen Alexy, a.a.O., S. 418) ersichtlich nicht vor einem Rivalen oder einer Rivalin schützt, liegt das Fallbeispiel völlig neben der Sache. Das prädestiniert es aber dafür, vielfach aufgegriffen und zitiert zu werden.

Das argumentum ad absurdum durch Weglassen des entscheidenden Merkmals „in Grundrechtsschutzgüter eingreifendes (Verhalten)“ hat sich in der einschlägigen Literatur seuchenartig verbreitet. Ich benenne hierfür nur ein weiteres Beispiel aus vielen: „Die Zurückführung allen erlaubten privaten Verhaltens (und dazu gehört auch das Sich am Kopf kratzen; J.S.) auf seine Zulassung durch die staatliche Rechtsordnung und seine umfassende Zurechnung zum Staat lassen es einerseits kaum noch zu, private Handlungen als von staatlicher Gewährung unabhängig, dem Staat insofern vorausliegend anzusehen und den Privaten als ihre freie Betätigung zuzurechnen. “(J. Hellermann, Die sogenannte negative Seite der Freiheitsrechte, Berlin 1993, S. 210). Goldrichtig, aber welcher Pappkamerad wird hier bekämpft? 
Private Handlungen, die Grundrechtsschutzgüter beeinträchtigen, muss sich jedoch der Staat wegen seiner Erlaubnis zurechnen lassen. ${ }^{18}$ Das ergibt sich aus ganz einfachen Überlegungen. Im Rechtsstaat kann die Zuordnung kollidierender Grundrechtsschutzgüter nicht von der Übermacht des Stärkeren abhängen, sondern nur von der rechtlichen Zuordnung durch den Staat. Zwei kollidierende Interessensphären sind abzugrenzen und dabei zwangsläufig im Kollisionsbereich zu beeinträchtigen. $\mathrm{Da}$ beide grundrechtlich geschützt sind, muss die staatliche Schlichtung durch ihr (Kollisions-) Recht auf die negatorischen Grundrechte beider Seiten Bedacht nehmen.

Das lässt sich am besten veranschaulichen bei einer Regelung der zulässigen Immissionen. Ich unterstelle, dass eine Zuführung von 50 Einheiten dem Emittierenden eine sinnvolle Nutzung seines Grundstücks und/oder Gewerbes erlaubt und dem Nachbarn ein gesundes Leben mit nur leichter Belästigung. Damit ist ein schonender Ausgleich, eine „praktische Konkordanz“ zwischen zwei Grundrechtssphären geschaffen. Was geschieht nun, wenn der Gesetzgeber den Wert von 50 auf 30 herabsetzt? Dann muss sich diese Maßnahme, ein Verbot der bislang 20 zusätzlichen Einheiten, an den Grundrechten des Emittenten messen lassen, selbstverständlich in ihrer abwehrrechtlichen Funktion. Wie steht es im umgekehrten Fall, wenn der Emissionswert auf 70 erhöht wird? Das soll nach Engländer kein staatlicher Eingriff sein. Dann bleiben zwei Lösungen übrig: Jeder Grundrechtsschutz entfällt; dazu bekennt sich niemand angesichts der Folgen, dass die fortan erlaubten 70 (oder evtl. auch 100) Einheiten vielen Menschen die Gesundheit kosten können. Also lässt man die Grundrechtsgeltung bestehen, aber eben nicht den - angeblich mangels Eingriffs irrelevanten - Abwehrmodus, sondern die grundrechtliche Schutzpflicht.

Nun ist es eine Binsenweisheit, dass die Grundrechte des status negativus auf die Abwehr staatlichen Handelns gerichtet sind, während die Schutzpflicht sich gegen staatliche Untätigkeit wendet und eine Schutzhandlung bewirken soll. Wogegen stellt sich nun der Nachbar bei einer Gesetzesnovelle, die dem Emittenten die Zuführung von zusätzlichen 20 Schadeinheiten erlaubt? Selbstverständlich gegen ein Handeln in Form der gesetzlichen Ermächtigung; diese soll unterlassen beziehungsweise wieder beseitigt werden. Engländer und die h.L. müssen aber auf dem Antrag beharren,

18 Ablehnend bspw. K. Seelmann, Grenzen privater Nothilfe, ZStW 89 (1977), S. 37 (57) mit (zu) einfachem Bestreiten: „Dagegen ist nicht zuzugeben, dass bei privater Notwehr und Nothilfe staatliche Machtausübung in dem Sinne vorliegt, dass das private Verteidigungshandeln dem Staat als eigenes Handeln zuzurechnen ist.“ 
dass der Beschwerdeführer die staatliche Unterlassung einer Schutzmaßnahme ${ }^{19}$ rügen und damit die Rücknahme der Novelle erwirken möchte.

Wenn das richtig wäre, müsste ein solcher verquerer Antrag auch im ersten Falle dem Emittenten zu empfehlen sein: Gegen das Verbot der bislang erlaubten 20 Einheiten wendet er sich dann mit dem Antrag, ihm durch Rücknahme der Gesetzesnovelle Schutz für seinen Gewerbebetrieb zu gewähren. Und gegen ein Berufsverbot würde man die staatliche Pflicht zum Schutz seiner Erwerbstätigkeit aktivieren mit dem erwünschten Ergebnis einer Unterlassung/Beseitigung des Verbots. ${ }^{20}$

2. Die unterschiedliche Grundrechtsgeltung für Gebote oder Verbote einerseits und Duldungsverpflichtungen andererseits ist leider allgemein üblich: ${ }^{21}$ Die erstgenannten werden den Grundrechten als Abwehrrechten konfrontiert, die letztgenannten sollen nur grundrechtlichen Schutzpflichten zuwiderlaufen. So hat es sich seit frühen spektakulären Leitentscheidungen des BVerfG eingebürgert und zwar bei Beeinträchtigungen, die ich in Parallele zu dem oben benutzten Beispiel als ideelle Immissionen bezeichnen möchte: Das Verbot gegen Lüth, den Regisseur Veit Harlan anzugreifen, war laut BVerfGE 7, 198 am abwehrrechtlichen Gehalt des Art. 5 I GG zu messen, die Verpflichtung des Blinkfüer-Verlags, den existenzbedrohenden Boykottaufruf Springers hinzunehmen, soll aber gemäß BVerfGE 25,256 nur die Missachtung einer staatlichen Grundrechtsschutzpflicht sein.

3. Engländer kritisiert an der Eingriffsbefugnis weiter: Bei meinem Konzept „fungierten die Abwehrrechte nicht mehr als Grenzen der Freiheitseinschränkungen, sondern als Schranken der Freiheitsgewährung. Das aber verkehrte den Zweck der grundrechtlichen Abwehrfunktion, nämlich die Freiheiten des Bürgers vor staatlichen Beeinträchtigungen zu schützen, in sein Gegenteil.“ (S. 181)

19 Die Zubilligung der 20 zusätzlichen Einheiten ist gegenüber dem Emittenten sowohl eine Erlaubnis wie auch ein Nichtverbieten. Bezeichnend für die Versuche, auf Biegen und Brechen auf eine grundrechtlich bedenkliche Unterlassung abzustellen, ist (für viele) Robbers, Sicherheit (Fn. 17), S. 129: „Selbst wenn man im Nicht- Verbieten eine Erlaubnis sehen wollte, werden die konkreten verletzenden oder gefährdenden Handlungen nicht solche des Staates. Zuzurechnen wäre allenfalls der Erfolg, aber nicht, weil der Staat selbst gehandelt hat, sondern weil er es unterlassen hat, seiner Schutzverpflichtung nachzukommen." Die Gesetzesnovelle war dann eben keine Handlung in Form der Ermächtigung, sondern eine Unterlassung (?).

20 Dazu auch J. Schwabe, Grundrechtlich begründete Pflichten des Staates zum Schutz gegen staatliche Bau- und Anlagengenehmigungen. Oder: Schuldet der Staat aktiven Schutz gegen sich selbst?, NVwZ 1983, 523.

Zustimmend L. Mahlberg, Gefahrenabwehr durch gewerbliche Sicherheitsunternehmen, Berlin 1983, S.117ff.

21 A.A. aber W. Cremer, Freiheitsgrundrechte, Tübingen 2003, S. 172 f. Weshalb Cremer im folgenden (S. 174 ff.) die Beseitigung des Erlaubnis-Eingriffs für unzureichend hält und sich mittels der Grundrechts-Schutzpflicht um eine zivilrechtliche Abwehrmöglichkeit sorgt, ist mir im Hinblick auf die seit über 100 Jahren verfügbaren Abwehr-Generalklauseln ( $\mathbb{S} 823$ analog und 1004 BGB) ganz unbegreiflich. 
Hier wird aus einer ganz schiefen Perspektive argumentiert, nämlich im Blick auf ein Zweierverhältnis Staat-Bürger, bei denen die Grundrechte in der Tat nur Freiheitseinschränkungen kontrollieren sollen und nicht Freiheitsgewährungen. In der hier vorliegenden Dreiecksbeziehung 22 des Staates mit zwei Bürgern gilt das natürlich überhaupt nicht. Wenn in dem obigen Fall 70 statt bislang 50 Emissionseinheiten erlaubt werden, dann fungieren die Grundrechte des Nachbarn als „Grenzen der Freiheitseinschränkung “ und gleichzeitig notwendigerweise bei dem Emittenten als Schranken der Freiheitsgewährung. Auch das ist ein banaler Effekt, dass jede Änderung einer Grenzziehung dem einen etwas wegnimmt und dem anderen etwas gewährt.

Nur ein verengter Blick auf die Zweierbeziehung kann auch zu der weit verbreiteten These führen, das Verhältnismäßigkeitsprinzip diene im öffentlichen Recht der Einschränkung der Staatsgewalt zu Gunsten des Bürgers, wenn es hingegen das Notwehrrecht einschränke, würde es genau umgekehrt die Freiheitsrechte des Bürgers reduzieren. ${ }^{23}$ Eine solche Auffassung bedingt, dass man unter Wegblenden des Angreifers ausschließlich auf den Angegriffenen schaut, während hier in Wahrheit das Verhältnismäßigkeitsprinzip für das Rechtsverhältnis zwischen Staat und Angreifer (auch ein „Bürger“) einzusetzen ist.

Die nachgerade grotesken Folgen erkennt man bei der Annahme, dass die Erfordernisse „geboten“ und „erforderlich“ aus $\$ 32$ entfernt würden, es also nach dem Gesetzeswortlaut erlaubt wäre, einen Angreifer niederzuschießen, den der Angegriffene auch mit einigen Fausthieben hätte verjagen können. Dann sollte diese staatliche Erlaubnis nicht am Grundsatz der Verhältnismäßigkeit kontrolliert werden, weil dadurch systemwidrig „die Freiheitsrechte des (angegriffenen) Bürgers reduziert“ würden?

\section{Zur Duldungspflicht}

Auch sie wird von Engländer als irrelevant bezeichnet.

1. Sie beziehe sich auf eine Bewehrung, und diese bedeute, „dass die Wahrnehmung der Erlaubnis durch bestimmte Verbote oder Gebote geschützt wird“. Das sei aber bei dem Notwehrrecht gar nicht der Fall. Dem Angreifer würden keine zusätzlichen Pflichten auferlegt, er dürfe der Bewehrung wegen „nicht weniger tun als zuvor“. „Die Erteilung der Befugnis zur Abwehr des Angriffs an den Verteidiger vermehrt nicht den Pflichtenkreis des Angreifers. Diesem bleiben dieselben Verletzungshandlungen untersagt wie bisher. “24

22 Zum mehrpoligen Verfassungsrechtsverhältnis J. Schwabe, Über Grundrechtsmythen, Abstraktionitis und Überproduktion in der Grundrechtsdogmatik, JZ 2007, S. 135.

23 U.a. H. Wagner, Individualistische oder überindividualistische Notwehrbegründung, Berlin 1984, S. 40, Fn.6 m.w. Nachw.; V. Krey, Zur Einschränkung des Notwehrrechts, JZ 1979, S. 713, Fn. 20.

24 Engländer, Nothilfe (Fn. 5), S. 182. 
Diese Argumentation wird schon bei einem flüchtigen Seitenblick von der Notwehr auf $\mathbb{S} 127$ I 1 StPO zunichte: Ohne diese Erlaubnis dürfte jedermann einen Privaten, der ihn festnehmen will, abwehren und verletzen, angesichts der staatlichen Ermächtigung darf er es nicht. Und auch bei der Notwehr ist es nicht anders, wie man leicht bei der Annahme erkennt, eine bislang geltende Einschränkung der Notwehrbefugnis (beispielsweise mit einem Verbot des Schusswaffeneinsatzes zum Schutz geringwertiger Vermögensgüter) würde aufgehoben. Zuvor durfte der Angreifer sich dann gegen den Schusswaffeneinsatz wehren, danach darf er es wegen seiner - neuen - Duldungspflicht prinzipiell nicht.

2. Den $\mathbb{S} 127$ StPO kann man auch als Lackmuspapier für die Argumentation von Alexy ${ }^{25}$ einsetzen, auf den sich Engländer auch bei der Duldungspflicht beruft. Seine Darlegungen, aufgehängt am Beispiel eines zum Passivrauchen Gezwungenen, können hier nicht im Einzelnen analysiert werden. Ihre Unschlüssigkeit wird aber schon deutlich, wenn man sie auf die obige und voll vergleichbare Konstellation überträgt, dass der Nachbar nach der Novellierung 70 Einheiten ertragen muss. Vollends stürzt das ganze Begründungsgebäude zusammen, sobald man es auf die Pflicht zur Duldung einer privaten Verhaftung wendet.

Alexy unterscheidet zwischen der Bewehrung der Duldungspflicht durch Verbote und Rechte des Eingreifenden und der staatlichen Durchsetzung der Duldungspflicht.

a) Zur Bewehrung heißt es, der Staat schütze die Freiheit (auch die aufgrund staatlicher Erlaubnis) gegen Widerstand. Gleichwohl müsse er sich die Freiheitsausübung (bei $\mathbb{S} 127$ StPO: durch vorläufige Festnahme) nicht zurechnen lassen. Begründung: Sonst müssten ihm unübersehbar viele Handlungen zugerechnet werden. Das erscheint mir unübersehbar dürftig.

b) Nicht besser steht es bei der Durchsetzung der staatlich auferlegten Duldungspflicht beispielsweise durch Strafen: „Der Staat mag bewirken, dass dem Nichtraucher (hier: dem Festgenommenen - J.S.) in bestimmten Situationen nur die Wahl bleibt, das Rauchen (hier: die Festnahme) hinzunehmen oder sich strafbar zu machen. Was hinzunehmen ist, bleibt aber eine private Handlung. “26

So einfach ist das also: Wenn statt eines Hoheitsträgers oder eines Beliehenen ein Privater mit staatlicher Ermächtigung und staatlicher Bestrafung der Gegenwehr einen anderen festnimmt, gelten die Grundrechte als Abwehrrechte nicht. Es fehlt offenbar an einem grundrechtsrelevanten Handlungs-Eingriff des Staates. Seine Erlaubnis und seine Durchsetzung der Duldungspflicht zählen nicht, solange der Zugriff durch zwei private Hände erfolgt.

26 Alexy, Grundrechte (Fn. 17) S. 419, ganz ähnlich Robbers, Sicherheit (Fn.17). 
Und zum Abschluss heißt es, wobei ich Raucher und Nichtraucher ausgetauscht habe gegen Festnehmender und Festgenommener: Eine Grundrechtsverletzung „kann nicht darin gesehen werden, dass der Staat als Beteiligter' an der Festnahme in das Grundrecht als Abwehrrecht eingreift, sondern nur darin, dass der Staat die Beziehungen zwischen Festnehmendem und Festgenommenen so regelt, dass er das Grundrecht des Festgenommenen in Form eines Schutzrechts verletzt. “27

Vollends bizarr wird diese Argumentation, wenn sich dem staatlichen Duldungszwang durch Strafandrohung noch die reale Duldungserzwingung hinzugesellt: Jemand „beschlagnahmt“ unter Berufung auf $\$ 904$ BGB eine fremde Sache. Der Gegenwehr des Besitzers begegnet der Zugriffsberechtigte aus Bequemlichkeit nicht mit eigener (staatlich erlaubter) Gewalt, er schaltet vielmehr die Polizei ein. Gleichwohl könnte dergleichen nach Alexy und der h.M. nie an Art.14 GG als Abwehrrecht gemessen werden, weil keine relevante Staatshandlung vorliegt, sondern nur ein privater Eingriff, gegen den der Staat keinen Schutz gewährt hat. Dass dieser private Eingriff gar nicht mal in der Wegnahme der Sache liegt, sondern nur in ihrer Empfangnahme von dem quasi vollstreckenden Polizisten sowie in der Sachnutzung, macht die betreffende Auffassung nochmal ein Stück verblüffender.

\section{Fazit}

Die Einwände gegen eine Geltung negatorischer Grundrechte gegenüber exzessiven Notwehrbefugnissen haben sich als nicht stichhaltig erwiesen. Falls nicht bessere gefunden werden, sollte sich die Strafrechtswissenschaft dieser These endlich öffnen.

\section{E. Von der Zoomaufnahme zum Weitwinkel}

Ein - kluger und deshalb zu berücksichtigender - Leser eines Entwurfs dieses Textes hat angemerkt: "Sie haben die Wirksamkeit der Grundrechte für das Strafrecht fokussiert. Nun stellen sich aber in Bezug auf das Verhältnis zum Zivilrecht letzten Endes dieselben Fragen. Sind die Antworten andere?"

Die Frage kann nur mit "nein" beantwortet werden. Sie könnte ausgelöst sein durch mindestens zwei Assoziationen:

- es gibt auch eine zivilrechtliche Notwehr

- Eingriffsermächtigungen und daran gekoppelte Duldungspflichten sind auch im Zivilrecht gang und gäbe.

Aber beide Prämissen stehen auf siechen Beinen.

Es ist selbstverständlich die in $\$ 227$ BGB normierte Notwehr nicht materiell Zivilrecht, weil sie in einem Zivilgesetzbuch steht, und die wortgleiche Regelung in $\$ 32$ StGB materiell öffentliches Recht (Untergruppe Strafrecht). Genauso unsinnig wäre 
ja die Vorstellung, bei der Heranziehung außerstrafrechtlicher Rechtfertigungsgründe, insbesondere aus dem BGB, würden diese von Zivilrecht in öffentliches Recht umgewandelt. Das erklärt sich ganz einfach aus folgendem:

Rechtfertigungsgründe sind Durchbrechungen von Verboten. Verbote jedoch, die, ebenso wie Gebote, sogenannte Primärnormen sind, lassen sich in den Dualismus öffentliches - privates Recht nicht einordnen. Das steht in Anbetracht dessen fest, dass niemand jemals auch nur versucht hat, ein den Umgang der Bürger betreffendes "Du sollst!" als öffentliches oder privates Recht zu qualifizieren. Dies ist erst bei den sogenannten Sekundärnormen möglich, die bestimmen, was bei einer Missachtung der Primärnorm zu geschehen hat: Eine (Folge-) Maßnahme von Behörden oder Richtern in Form einer Verfügung oder einer Sanktion ist öffentlichrechtlich geregelt (weil sie besonders Verhalten von Hoheitsträgern normiert). Eine Maßnahme von Privaten (Kündigung, Unterlassungs- oder Entschädigungsverlangen) ist, weil "Jedermann-Verhalten" betreffend, zivilrechtlich geregelt. Bei den Sekundärnormen knüpfen dann die unterschiedlichen Rechtsfolgen für das öffentliche oder das private Recht an: Verfahren samt Vollstreckung, Entschädigung, Gerichtsbarkeit und anderes.

Was für die Primärnorm gilt, trifft logischerweise auch für ihre Durchbrechung durch Rechtfertigungsgründe zu. Wenn das "Du sollst!" einer Zuordnung zu den Alternativen öffentliches und privates Recht nicht zugänglich ist, dann selbstverständlich auch nicht ein "außer, wenn..." Die universal wirkenden Rechtfertigungsgründe sind in öffentlichrechtlichen oder privatrechtlichen Kodifikationen auffindbar, aber weder öffentlich-rechtlicher noch zivilrechtlicher Natur.

Ganz parallel verhält es sich bei allen Wechselspielen zwischen bestimmten Verboten und den angelagerten Erlaubnissen. Konkret: Die oben angenommene Regelung einer zulässigen Emissionsbelastung mit 50 Einheiten bedeutet für den Emittenten eine Erlaubnis zur Zuführung dieser Menge, die ein denkbares Totalverbot aus dem Eigentum des Nachbarn ausschließt und diesem eine Duldungspflicht auferlegt. Die Einheiten 50 - 70 sind dem Emittenten verboten, und dem Nachbarn alle Abwehrmaßnahmen gegen eine 40er Immission.

Diese Regulierung durch verbietende Primärnormen und ihre zwangsläufige Verkoppelung durch benachbarte Erlaubnisse kann nach freiem Belieben in das BGB oder in ein Umweltgesetzbuch geschrieben werden. Letzteres liegt näher, weil man sie nur dort mit den öffentlich-rechtlichen Sekundärnormen in Form behördlicher Befugnisse verknüpfen kann (und bei den zivilrechtlichen Sekundärnormen, der Anspruchsbegründung, auf die Verletzung des (Umwelt-) Schutzgesetzes zurückgreift). Aber damit werden die Grenzwerte nicht zu öffentlichem Recht, während sie bei ihrer ebenso möglichen Platzierung im BGB Zivilrechtsnormen wären. 
Ergebnis: Stets geht es um die Grundrechtsverträglichkeit belastender Primärnormen, und zwar ausschließlich deshalb, weil das Verbot (oder ein Duldungsgebot) belastet und die Frage, ob es durch Verwaltungsakt, durch ein Strafurteil oder privatrechtliche Ansprüche, also mittels welcher Sekundärnormen, durchgesetzt wird, ohne jedes Interesse ist.

Die Frage nach der Geltung der Grundrechte "im Privatrecht" ist folglich unsinnig. 